\title{
Temperature Manipulation in Songbird Brain Implicates the Premotor Nucleus HVC in Birdsong Syntax
}

\author{
Yisi S. Zhang, ${ }^{1}$ Jason D. Wittenbach, ${ }^{1}$ DDezhe Z. Jin, ${ }^{1,3}$ and Alexay A. Kozhevnikov ${ }^{1,2}$ \\ ${ }^{1}$ Department of Physics, ${ }^{2}$ Department of Psychology, and ${ }^{3}$ Center for Neural Engineering, Pennsylvania State University, University Park, Pennsylvania \\ 16802
}

Variable motor sequences of animals are often structured and can be described by probabilistic transition rules between action elements. Examples include the songs of many songbird species such as the Bengalese finch, which consist of stereotypical syllables sequenced according to probabilistic rules (song syntax). The neural mechanisms behind such rules are poorly understood. Here, we investigate where the song syntax is encoded in the brain of the Bengalese finch by rapidly and reversibly manipulating the temperature in the song production pathway. Cooling the premotor nucleus HVC (proper name) slows down the song tempo, consistent with the idea that HVC controls moment-to-moment timings of acoustic features in the syllables. More importantly, cooling HVC alters the transition probabilities between syllables. Cooling HVC reduces the number of repetitions of long-repeated syllables and increases the randomness of syllable sequences. In contrast, cooling the downstream motor area RA (robust nucleus of the acropallium), which is critical for singing, does not affect the song syntax. Unilateral cooling of HVC shows that control of syllables is mostly lateralized to the left HVC, whereas transition probabilities between the syllables can be affected by cooling HVC in either hemisphere to varying degrees. These results show that HVC is a key site for encoding song syntax in the Bengalese finch. HVC is thus involved both in encoding timings within syllables and in sequencing probabilistic transitions between syllables. Our finding suggests that probabilistic selections and fine-grained timings of action elements can be integrated within the same neural circuits.

Key words: Bengalese finch; birdsong; birdsong syntax; cooling; HVC; lateralization

\section{Significance Statement}

Many animal behaviors such as birdsong consist of variable sequences of discrete actions. Where and how the probabilistic rules of such sequences are encoded in the brain is poorly understood. We locally and reversibly cooled brain areas in songbirds during singing. Mild cooling of area HVC in the Bengalese finch brain-a premotor area homologous to the mammalian premotor cortex-alters the statistics of the syllable sequences, suggesting that HVC is critical for birdsong sequences. HVC is also known for controlling moment-to-moment timings within syllables. Our results show that timing and probabilistic sequencing of actions can share the same neural circuits in local brain areas.

\section{Introduction}

Variable sequences of discrete actions are prevalent in animal and human behaviors. Examples include human speech, birdsong (Doupe and Kuhl, 1999), whale song (Payne and McVay, 1971),

Received June 7, 2016; revised Jan. 3, 2017; accepted Jan. 17, 2017.

Author contributions: D.Z.J. and A.A.K. designed research; Y.S.Z. performed research; Y.S.Z. and J.D.W. analyzed data; Y.S.Z., D.Z.J., and A.A.K. wrote the paper.

This work was supported by the National Science Foundation (Grant 0827731), the Pennsylvania State University Department of Physics, and the Huck Institute for Life Sciences. We thank Guerau Cabrera and Bruce Langford for assistance at the initial stage of the project and Dmitry Aronov for technical advice.

The authors declare no competing financial interests.

Y.S. Zhang's present address: Princeton Neuroscience Institute, Princeton, NJ 08544.

J.D. Wittenbach's present address: Janelia Research Campus, Ashburn, VA 20147.

Correspondence should be addressed to Dezhe Z. Jin, Department of Physics, Pennsylvania State University, 104 Davey Laboratory, PMB 206, University Park, PA 16802. E-mail: dzj2@psu.edu.

DOI:10.1523/JNEUROSCI.1827-16.2017

Copyright $\odot 2017$ the authors $\quad 0270-6474 / 17 / 372600-12 \$ 15.00 / 0$ and grooming in rodents (Cromwell and Berridge, 1996). These behavioral sequences display regularities and structures that are often referred to as "action syntax" (Lashley, 1951), in analogy to syntax in language (Chomsky, 1965). Where and how such syntactic rules for actions are encoded in the brain remains an unsolved problem.

The songbird is a model system for studying the neural mechanisms of vocal sequences (Doupe and Kuhl, 1999). Many songbird species sing songs with variable sequences (Okanoya, 2004). One often-studied species is the Bengalese finch, the song of which consists of variable sequences of stereotyped vocal elements called syllables (Fig. 1A, B; Okanoya and Yamaguchi, 1997; Woolley and Rubel, 1997; Jin and Kozhevnikov, 2011). Certain statistical properties of the song, such as repeat distributions (the probability of observing a syllable a certain number of times in a row) and pairwise 

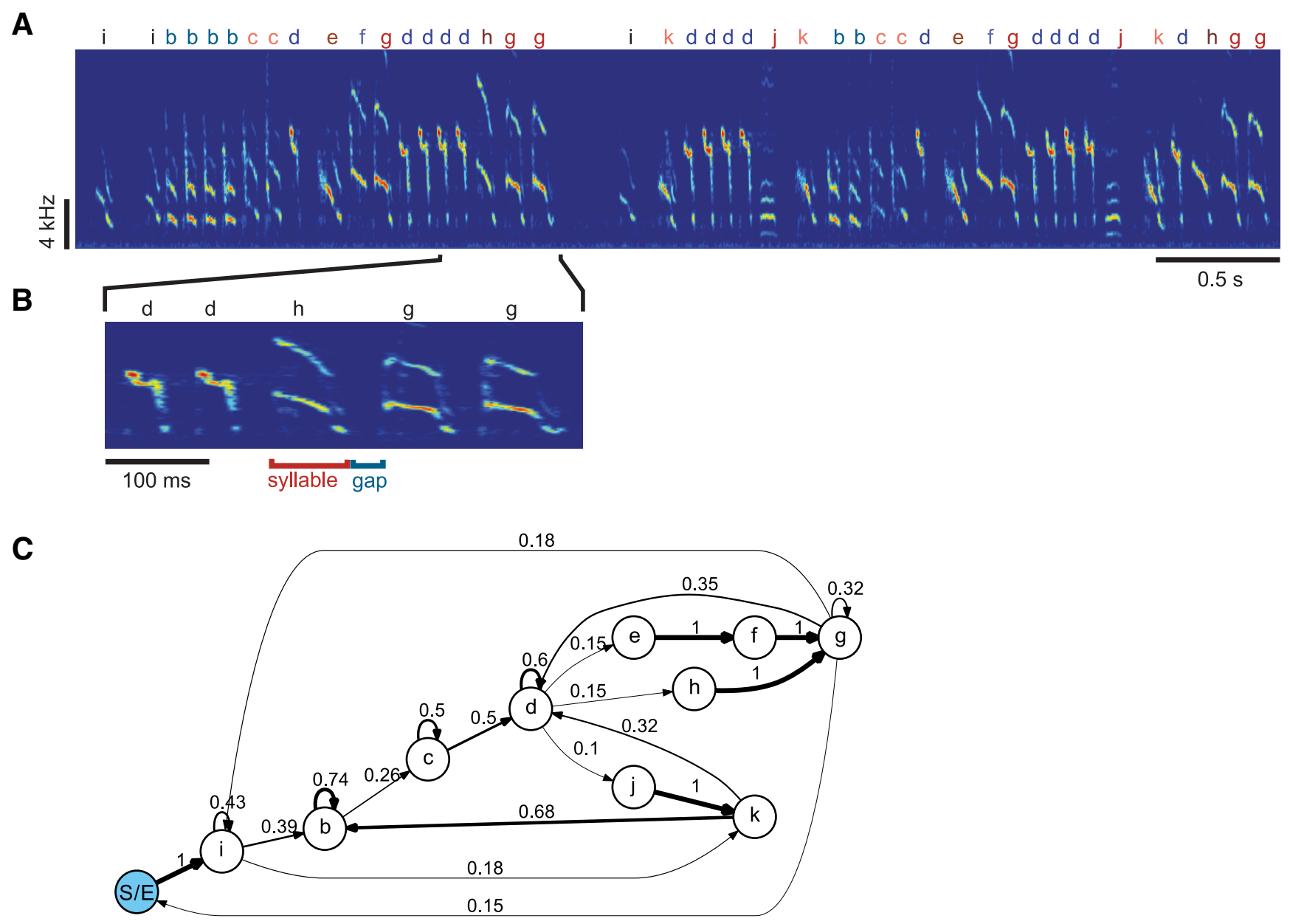

Figure 1. Structure of Bengalese finch song. A, A song bout consists of a sequence of song syllables (labeled with letters " $\mathrm{a}$ " through " $\mathrm{k}$ " above the spectrogram). $\boldsymbol{B}$, Syllables are separated by silent gaps. $C$, Song syntax can be visualized by a transition diagram. Arrows show the allowed transitions between the syllables and the numbers are the transition probabilities.

transition probabilities (the probability of observing one syllable after another; Fig. 1C), are stable over long periods of time (Warren et al., 2012). Statistical rules describing patterns such as these comprise the song syntax (Okanoya, 2004).

It has been established that the premotor nucleus HVC (proper name) plays a role in controlling the tempo of singing in songbirds (Hahnloser et al., 2002; Long and Fee, 2008; Long et al., 2010). However, the role of HVC in song syntax remains unclear. One idea is that the probabilistic syllable selection is determined within HVC (Jin, 2009; Hanuschkin et al., 2011; Wittenbach et al., 2015). This mechanism is built on the notion that syllables are encoded with chain networks of HVC neurons projecting to the premotor nucleus RA (robust nucleus of the arcopallium; Fee et al., 2004; Long et al., 2010). As the activity propagates along such a syllable chain, $H_{V} C_{R A}$ neurons burst once at precise times and drive RA neurons to generate moment-to-moment acoustic features (Fee et al., 2004). When the activity reaches the end of the syllable chain, it briefly activates a number of syllable chains that are connected. Among them, the next syllable chain to propagate spikes is selected stochastically via a winner-take-all mechanism mediated through HVC interneurons, completing the probabilistic syllable transition (Chang and Jin, 2009; Jin, 2009). An alternative idea is that sequences are determined, not in HVC, but in areas upstream of HVC; lesion studies suggested the thalamic nucleus Uva (nucleus uvaeformis; Williams and Vicario, 1993) or NIf (nucleus interface of the nidopallium; Hosino and Okanoya, 2000). This proposal fits with the general scheme of hierarchical organization of motor sequence control (Rosenbaum et al., 1983; Yu and Margoliash, 1996). A third idea is that song is produced via activity propagating around a feedback loop consisting of HVC, RA, the brainstem, and Uva (Wild, 1997; Schmidt, 2003; Ashmore et al., 2005), with this loop generating $\mathrm{HVC}_{\mathrm{RA}}$ bursts (Gibb et al., 2009; Hamaguchi et al., 2016). In this model, any of the nuclei in the loop could determine syllable sequences.

To evaluate the role of HVC in song syntax, we reversibly cooled HVC as a means of perturbing its dynamics in singing Bengalese finches. For comparison, we also cooled RA, the immediate target of HVC in the song pathway. Previously cooling has been used on the zebra finch (Long and Fee, 2008; Aronov and Fee, 2011; Hamaguchi et al., 2016) and the canary (Goldin et al., 2013) to investigate how different brain regions contribute to the control of song timing. Here, we applied the same technique to understand how song syntax is encoded. We found that cooling HVC systematically affects the transition probabilities between the syllables. In contrast, cooling RA produced minimal effects. Our observations show that HVC is a key area for shaping the song syntax of the Bengalese 
finch. HVC is thus critical for both moment-to-moment timings within syllables and probabilistic sequencing of syllables.

\section{Materials and Methods}

\section{Subjects}

Eight male Bengalese finches (Lonchura striata domestica, $>120 \mathrm{~d}$ after hatching) were used in the experiments. During the experiments, birds were housed individually in soundattenuating chambers on a $14 \mathrm{~h} / 10 \mathrm{~h}$ light/dark photoperiod. Food and water were provided $a d$ libitum except for the night before surgery. All procedures were performed in accordance with the protocol approved by the local institutional animal care and use committee.

\section{Reversible cooling}

We constructed a Peltier device for reversible cooling or heating of the songbird brain. The device used a convection air-cooled heat sink and was similar to one described previously (Aronov and Fee, 2011). Two Peltier modules (12-0.45-1.3; TE Technology) sharing the same heat sink were connected in series and each had a gold-plated silver contact pad attached to the cold side. Jumper connectors (A9577-001; Omnetics) were used to switch between bilateral and unilateral modes of operation (Fig. $2 A$ ). The cooling pads were placed bilaterally on the brain surface on top of HVC (Fig. 2A). The device was attached to the skull using dental acrylic. The temperature of the two cooling pads was changed bilaterally or unilaterally. A calibration of HVC temperature as a function of the current applied to the Peltier module of the device was performed. The device was capable of altering $\mathrm{HVC}$ temperature by $\sim 5^{\circ} \mathrm{C}$ in either direction (Fig. $2 B$ ). Manipulating the temperature of one hemisphere did not affect the other hemisphere (Fig. 2B). The device was connected to a rotary commutator with a flexible cable, which allowed the bird to move freely. Temperature in HVC was monitored with a miniature thermocouple (5SRTC-TT-K40-36; Omega) inserted in the vicinity of HVC at the depth of $0.5 \mathrm{~mm}$. We also made a probe attached to the device for cooling RA, as was done in previous studies for cooling deeper brain areas (Long and Fee, 2008). The cooling probes were implanted bilaterally into RA. To measure RA temperature, a thermocouple was inserted anterior to the probe to reach RA from a different angle. Another thermocouple was inserted in the ipsilateral HVC at a depth of $0.5 \mathrm{~mm}$ beneath the brain surface. Both RA and HVC temperatures were recorded simultaneously while applying currents to the device.

\section{Data collection}

Undirected songs were recorded with an omnidirectional microphone (Cardioid Pro 44; Audio-Technica) during temperature manipulations. The audio signal was amplified (DMP3; M-Audio), band-pass filtered (0.3-10 $\mathrm{kHz}$ ), digitized with a 16-bit A/D card (PCI-6251; , National Instrument), and recorded using customized song-triggered software written in MATLAB (The MathWorks) at a sampling rate of $40 \mathrm{kHz}$. Song recording was stopped when the postsong silence exceeded $2 \mathrm{~s}$. Five to seven different current settings were used in each bird, ranging from $-75 \mathrm{~mA}$ to $200 \mathrm{~mA}$, corresponding to temperature changes from $\sim 2.0^{\circ} \mathrm{C}$ to $\sim-4.5^{\circ} \mathrm{C}$ relative to the brain temperature measured in the normal condition $\left(40.3 \pm 1.7^{\circ} \mathrm{C}\right.$ during the waking hours). Whenever current was adjusted, we allowed $10 \mathrm{~min}$ for the temperature to stabilize. A set of experimental conditions consisted of two to three temperature conditions interleaved with normal conditions. This set was repeated every $2-3 \mathrm{~d}$.
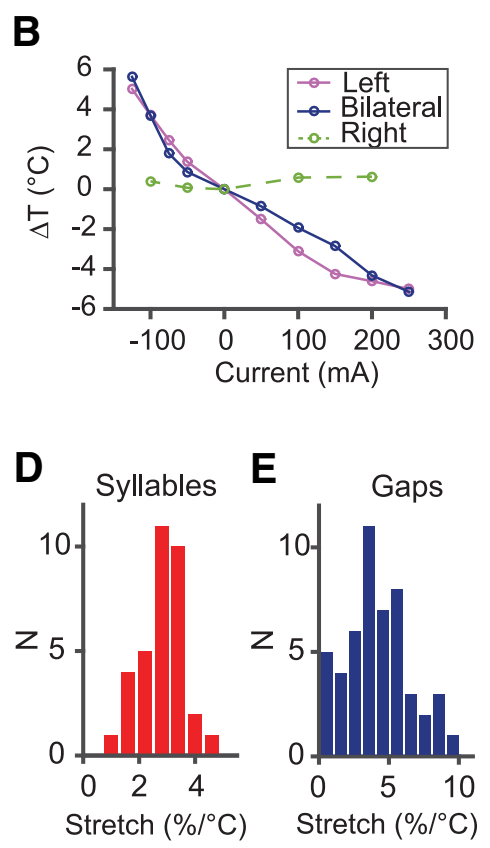

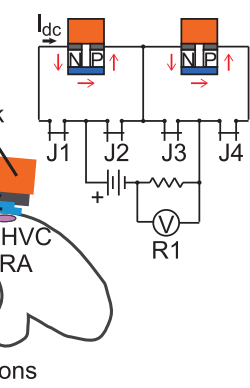

. HVC temperature manipulation. A, Setup of the experiment. The contact pad is placed on the surface of the brain and consisting of four syllables is shown at different HVC temperatures aligned to the onset of the first syllable. $D, E$, Histograms of the fractional temperature-induced duration changes of all syllables (left) and gaps (right) in our dataset. Syllable durations are stretched by $2.8 \pm 0.9 \% /{ }^{\circ} \mathrm{C}($ mean $\pm S D, n=34)$ and gap durations are stretched by $4.2 \pm 2.5 \% /{ }^{\circ} \mathrm{C}(n=50)$.

Segmentation of the song into syllables and intersyllable gaps was done offline based on the envelope of the sound amplitude crossing a threshold. Syllable identification was first performed automatically using a custom sorting algorithm (Jin and Kozhevnikov, 2011). The results of automatic sorting were verified manually by visual inspection of the syllable spectrograms. The end of a song in a continuous recording was identified by the appearance of a silent period longer than $2 \mathrm{~s}$ or an introductory note. Introductory notes were excluded from analysis.

\section{Data analysis}

Syllable and gap durations. We compared the durations of the song syllables and the intersyllable gaps under the normal condition and when HVC or RA temperature was manipulated. To eliminate possible variation of the song over days, the comparison was done between data collected on the same day. At each temperature, the mean duration of each syllable or gap was computed. To compute the change of the mean durations with temperature, we fit a weighted linear regression model of the mean duration as a function of change in temperature. The weight at each temperature was the inverse square of the SD of the durations measured at that temperature. The fractional stretch of the duration with cooling, denoted as $d D_{\mathrm{T}}$, was obtained by dividing the slope of the line by the mean duration in the normal condition. The fractional stretch is expressed in percent per degree Celsius.

Syllable repetitions. For each repeated syllable, the effect of temperature on the length of repetitions was quantified with $d R_{\mathrm{T}}$, which is the slope of a weighted linear regression model of the mean repetition length as a function of the temperature. Based on the cooling effects, we defined two classes of repeated syllables, denoted type I (with positive $d R_{\mathrm{T}}$ ) and type II (with zero $d R_{\mathrm{T}}$ ). To define these two classes rigorously, we fit the distribution of $d R_{\mathrm{T}}$ values across repeated syllables with a mixture of two Gaussians using the function mle in MATLAB for maximum likelihood estimation. To determine whether the two types could be distinguished 
by the variability of the repetition lengths, we trained a linear classifier using linear discriminant analysis (McLachlan, 2004) to predict type I versus type II based on the SD of the repetition length distribution in the normal condition. We quantified the goodness of fit by the misclassification rate via leave-one-out cross-validation. One syllable was near the border and was classified as type I based on temperature sensitivity but as type II based on SD of repetition distribution in the normal condition. To keep the criterion consistent across analyses, we classified it as type II for subsequent analyses. Some long-repeating syllables had a bimodal distribution of repetition number, with a sharp peak at one to two repetitions and a broad peak with a long tail (Wittenbach et al., 2015). This has been suggested to be a "many-to-one mapping" from multiple neural representations to syllables sharing similar acoustic features (Jin and Kozhevnikov, 2011). For these syllables, we discarded the repetitions with lengths that were $\leq 2$ and used only the broad part of the distribution.

Branch points. A branch point in the song syntax is where a syllable transitions probabilistically to two or more other syllables. To compute the transition probability $p_{\mathrm{ij}}$ from syllable $i$ to syllable $j(i \neq j)$, we counted the number of transitions from syllable $i$ to $j$ and divided it by the total number of transitions from syllable $i$. This creates a matrix of transition probabilities, the rows of which sum to 1 . In analyzing the branching points, segments of repeating syllables are treated as a single "repeated syllable." Therefore, the diagonal elements of the transition matrix were set to zero. Branch points were identified under the normal condition. To account for the possibility of misidentification of syllables due to ambiguity, we excluded transitions with probabilities below a noise threshold (see below). In the cooling/heating conditions, we kept the transitions with probabilities below the noise level if the transitions existed in the normal condition. This enabled us to monitor the changes of the transition probabilities across all conditions.

We used a fairly conservative procedure for estimating the noise level of the transition probabilities. We estimated the rate of ambiguous syllables as follows. We randomly chose 10 songs under the most cooled condition for each bird and labeled the syllables that did not clearly belong to any type. We reasoned that more ambiguity arises due to more deformations of acoustic structures in these conditions and we should obtain an upper bound for the error rates. The error rate of syllable identification for each bird is $p_{\mathrm{e}}=N_{\mathrm{e}} / N$, where $N_{\mathrm{e}}$ and $N$ are the number of ambiguous syllables and the total number of syllables, respectively. $p_{\mathrm{e}}$ can fluctuate if a different set of $N$ syllables were observed. Because such sampling leads to a binomial distribution, we estimated the upper $95 \%$ confidence level for $p_{\mathrm{e}}$ using the Wilson score interval with continuity correction (Newcombe, 1998), given by the following:

$$
w^{+}=\frac{2 N p_{e}+z^{2}+\left(1+z \sqrt{z^{2}-\frac{1}{N}+4 N p_{e}\left(1-p_{e}\right)-\left(4 p_{e}-2\right)}\right)}{2\left(N+z^{2}\right)} .
$$

where $z=1.96$. If $w^{+}>1$, it was set to $w^{+}=1$.

For a syllable, $i$, the probability of misidentification is $P($ error $\mid i)$ and the total count of incorrectly identified syllables is $e_{\mathrm{i}}=P(\operatorname{error} \mid i) N_{\mathrm{i}}$, where $N_{\mathrm{i}}$ is total number of counts of syllable $i$. We assumed that, in the transition $i \rightarrow j$, the case of both syllables being misidentified is negligible and a false detection of this transition is due to either a misidentified $i$ or $j$, but not both. We further assumed that occurrence and misidentification are independent; therefore, the number of incorrect $i \rightarrow j$ transitions is given by the following:

$$
e_{i j}=e_{i} P_{j}+e_{j} P_{i}=P(\text { error } \mid i) N_{i} P_{j}+P(\text { error } \mid j) N_{j} P_{i},
$$

where $P_{\mathrm{i}}=N_{\mathrm{i}} / N$ is the probability of syllable $i$ in the song. Bayes theorem leads to $P($ error $\mid i)=P(i \mid$ error $) p_{e} / P_{i}$ and $P($ error $\mid j)=P(j \mid$ error) $p_{e} / P_{j}$ and the following:

$$
e_{i j}=P(i \mid \text { error }) p_{e} N_{j}+P(j \mid \text { error }) p_{e} N_{i} \leq p_{e}\left(N_{i}+N_{j}\right) .
$$

Therefore, a conservative estimate of the number of incorrect $i \rightarrow j$ transitions due to ambiguity is the error rate multiplied by the total counts of syllables $i$ and $j$. With a confidence level of $95 \%$, we have $p_{\mathrm{e}} \leq$ $w^{+}$, so we set the noise threshold for the $i \rightarrow j$ transition to the following:

$$
\theta_{i j}=w^{+}\left(N_{i}+N_{j}\right)
$$

For five birds, the error rate was $1.73 \pm 0.64 \%$ when cooled by $4^{\circ} \mathrm{C}$ (the most cooled condition) and $0.52 \pm 0.18 \%$ in the normal condition. We used the first value to calculate the noise threshold in the cooling conditions and the second to calculate the noise threshold in the normal and the warming conditions.

We used transition entropy, defined as $H_{i}=-\Sigma_{j} p_{i j} \log _{2} p_{i j}$, to quantify the variability of the transitions at a branch point of syllable $i$ (Okanoya and Yamaguchi, 1997; Woolley and Rubel, 1997; Sakata and Brainard, 2009). The transition entropy was normalized by the maximum possible value of entropy for a branch point with $n_{\mathrm{i}}$ possible transitions, which is $\log _{2} n_{\mathrm{i}}$.

Song bouts. The end of a song bout was identified as a silent period $>2$ s or one followed by introductory notes. The significance of the change in the number of syllables in a bout with the temperature was assessed with the linear mixed model (LMM; see below), treating the subjects as a random factor. The entropy of the probability distribution of the ending syllables was calculated as $H_{e}=-\Sigma_{i} p_{i e} \log _{2} p_{i e}$, in which $p_{\mathrm{ie}}$ is the probability for a song bout to be end after syllable $i$. The rate of song production was quantified by the time elapsed between two consecutive song bouts.

Lateralization. The contributions of left and right HVCs to syllable repetition were assessed by comparing the effect of temperature on repetition length, $d R_{\mathrm{T}}$, between unilateral and bilateral cooling. To evaluate the lateralization of a syllable repetition, we first chose the side with the larger $d R_{\mathrm{T}}$ in unilateral cooling and then compared it with $d R_{\mathrm{T}}$ in the bilateral cooling. Hemispheric contributions to transition probabilities at branch points were assessed by comparing changes of the transition entropy between unilateral and bilateral cooling.

\section{Tests for statistical significance}

The criterion for significance in all tests was set at $\alpha=0.05$.

Syllable repetitions. We used an LMM (Gelman and Hill, 2006) to analyze the relationship between the mean repetition length, $R$, and the change of temperature of either HVC or RA from the normal condition, $\Delta T$. We allowed for the possibility that the slope and the intercept of the relationship can vary from syllables to syllable. The equation for the LMM is as follows:

$$
R_{i j}=\left(\beta_{0}+s_{j o}\right)+\left(\beta_{1}+s_{j 1}\right) \Delta T_{i}+\epsilon .
$$

Where the first index denotes the temperature condition and the second denotes the syllable identity. $R_{\mathrm{ij}}$ is the mean repetition length for the $i$-th condition and the $j$-th syllable; $\Delta T_{\mathrm{i}}$ is the temperature change for the $i$-th condition; $\beta_{0}$ (fixed effect) is the mean intercept across syllables; $s_{\mathrm{j} 0}$ (random effect) is the shift in the intercept for the $j$-th syllable; $\beta_{1}$ (fixed effect) is the average slope across syllables; $s_{j 1}$ (random effect) is the shift in the slope for the $j$-th syllable; $\epsilon$ is the random error. The LMM analysis was performed using the fitlme function in MATLAB with the formula $R \sim 1+\Delta T+(1 \mid$ syllable label $)+(\Delta T \mid$ syllable label $)$. We tested whether the coefficient $\beta_{1}$ was significantly different from zero. $\beta_{1}$ was estimated by the MATLAB implementation of maximum likelihood estimation and the $p$-value was given in the MATLAB output.

To determine whether HVC cooling affected the type I and type II repeated syllables differently, we introduced a dummy variable, $C$, for type I/II syllables and tested for an interaction between $\mathrm{C}$ and $\Delta T$. In addition, we estimated the random effect of the syllable labels. The model was as follows:

$$
R_{i j}=\beta_{0}=\beta_{1} \Delta T_{i j}+\beta_{2} C_{i j}+\beta_{3} \Delta T_{i j} C_{i j}+s_{j}+\epsilon .
$$

We used MATLAB function fitlme with the formula $R \sim 1+\Delta T+\mathrm{C}+$ $\Delta T: C+(1 \mid$ syallable label $)$ to test whether the interaction coefficient $\beta_{3}$ was different from zero. The $p$-value was reported in the MATLAB statistical outputs. The comparison of HVC and RA cooling on syllable repetitions was performed using the same model by substituting type I/II with HVC/RA cooling when defining the dummy variable, $C$.

Null model for temperature effects. A simple model attributes the cooling effects of repetition entirely to the stretches of the syllables and the gaps. There could be a fixed duration of time during which repetitions 
occur that is not affected by temperature changes. Longer syllables at cooler temperatures would then lead to fewer repetitions. Let $L$ be the duration of a repeat bout. Because the syllables and gaps stretch linearly with temperature change, $\Delta T$, the duration of one syllable plus gap can be expressed as $D=D_{0}\left(1-d D_{T} \cdot \Delta T\right)$, where $D_{0}$ is the duration in the normal condition and $d D_{\mathrm{T}}$ is the fractional change in duration per unit change in temperature. The number of repetitions is then given by $\mathrm{N}(\Delta \mathrm{T})=\frac{\mathrm{L}}{\mathrm{D}(\Delta \mathrm{T})}=\frac{\mathrm{N}(0)}{1-\mathrm{dD}_{\mathrm{T}} \cdot \Delta \mathrm{T}}$. Here, $N(0)$ is the number of repetitions in the normal condition. Therefore, the model predicts that the repeat distribution at $\Delta T$ can be generated directly from that in the normal condition through rescaling by a factor of $1 /\left(1-\mathrm{dD}_{\mathrm{T}} \cdot \Delta \mathrm{T}\right)$. The same scaling will then hold for the means of the distributions. To generate model predictions for how the mean number of repetitions will change as a function of $\Delta T$, we set $d D_{\mathrm{T}}$ to the average between the experimentally observed values of this quantity for syllables and gaps. To assess the difference between the null model predictions and our actual observations, we compared $d R_{\mathrm{T}}$ from the model and the data. We centered the data by subtracting the mean repetition length at $\Delta T=0$ for each syllable. To test whether the $d R_{\mathrm{T}}$ was the same for model and data, we applied an analysis of covariance (ANCOVA) using the MATLAB implementation aoctool to fit a separate line for each group. The significance was given based on an $F$ test between deviations of $d R_{\mathrm{T}}$ from the mean for the model and the data.

Branch points. The significance of changes in transition probabilities for each branch point were assessed with a $\chi^{2}$ test for independence or Fisher's exact test if some counts of transitions were smaller than 10 (Fisher, 1922). For the transitions from each syllable, a contingency table was constructed by counting the number of transitions to all target syllables. Each row of the table represents one target syllable and each column represents a different condition. For testing the effect of temperature, each column contained the counts to the target syllables at a different temperature. For testing day-to-day fluctuations, each column contained the counts observed from a different day. Such tables were used to test the null hypothesis that the transition probabilities remain the same across different conditions.

The significance that the transition entropy changed with temperature was assessed using a two-tailed $t$ test on the null hypothesis that a linear regression of entropy as a function of temperature change results in a slope of zero. To assess the dependence of this slope, $\beta$, on the transition entropy at $\Delta T=0, H_{0}$, we performed two-tailed $t$ tests on whether the slope of $\beta$ versus $H_{0}$ was different from 0 .

RA cooling and the syllable and gap durations. While cooling RA, HVC temperature changed by $\sim 32 \%$ of the temperature change in RA. To determine whether changes in syllable (or gap) duration observed during RA temperature manipulation could be explained by the collateral cooling in $\mathrm{HVC}$, we tested the null hypothesis that the fractional stretch of the syllables (or gaps) per unit temperature change in RA is equal to $32 \%$ of that observed during HVC cooling. We compared the fractional change of syllable (or gap) durations during RA cooling with those produced by HVC cooling multiplied by a factor of 0.32 . The significance of the difference in means between the two groups was evaluated with a two-tailed $t$ test.

Hemispheric asymmetry. To assess the significance of the correlation between the gap stretches under left versus right HVC cooling, we performed a shuffle test in which we randomized the assignment of gap pairs to the two conditions. The randomly paired gaps were surrogates for the null hypothesis of uncorrelated effects on the same gaps from cooling the left versus the right HVC. The $p$-value for the correlation coefficient was calculated from the percentile of the observed correlation coefficient in the shuffle distribution. To compare left, right, and bilateral HVC cooling effects on repetition length and transition entropy, we performed an ANCOVA on the slopes of these values with respect to temperature change using the MATLAB routine aoctool. If the between-group difference was significant, we performed a TukeyKramer post hoc test using MATLAB multcompare to find out which groups contributed to the difference.

\section{Results}

We manipulated the temperature of HVC in adult Bengalese finches. Relative to the normal condition, temperature changes in $\mathrm{HVC}$ ranged from $\sim-4.5^{\circ} \mathrm{C}$ (coolest) to $\sim 2.0^{\circ} \mathrm{C}$ (warmest). To simplify the presentation, we will mainly present the cooling effects and leave the heating effects to the figures. Unless specified, manipulations are bilateral and the temperatures in the two hemispheres are kept the same.

\section{Syllable and gap durations}

Cooling stretched the songs of the Bengalese finch (Fig. 2C), similar to previous studies that have cooled HVC in the zebra finch (Long and Fee, 2008). The durations of song syllables were stretched by $2.8 \pm 0.9 \% /{ }^{\circ} \mathrm{C}$ (mean $\pm \mathrm{SD}$, percentage per degree Celsius, $n=34$ syllables in 5 birds; Fig. $2 D$ ). The durations of intersyllable gaps were stretched by $4.2 \pm 2.5 \% /{ }^{\circ} \mathrm{C}(n=50$ intersyllable gaps in 5 birds; Fig. $2 E$ ). The stretch in gap durations was greater than that in syllable durations $\left(p=7.3 \times 10^{-4}\right.$, twotailed $t$ test). Overall, the amount of stretch in both the syllable and gap durations was comparable to that seen in cooling HVC in the zebra finch (Long and Fee, 2008). Heating had the opposite effects (Fig. 2C).

\section{Cooling HVC changes the song syntax}

\section{Syllable repetitions}

Syllable repetitions are a common feature of Bengalese finch song (Jin and Kozhevnikov, 2011; Wittenbach et al., 2015). Cooling HVC consistently reduced the number of repetitions within a repeat bout while heating increased it, especially for those syllables with long and variable repetition lengths. Figure $3, A$ and $B$, shows two examples. For the long-repeated syllable "A" of Bird 1, the distribution of repetition lengths shifted toward fewer repetitions as HVC was cooled (Fig. 3B). In contrast, the shortrepeating syllable " $C$ " of Bird 2 was not affected by changes in temperature (Fig. $3 B$ ). We quantified this effect with the slope of the mean repetition length with respect to changes of HVC temperature, $d R_{\mathrm{T}}$ (Fig. 3C). Across all repeated syllables, $d R_{\mathrm{T}}$ had a bimodal distribution, with one subset (type I) strongly affected by HVC temperature, whereas the other subset (type II) remained unaffected (Fig. 3D).

We next investigated whether these two subsets had distinctive repetition length distributions in the normal condition. We observed that variability of repetition length, defined as the SD of repetition length in the normal condition, was positively correlated with the temperature effect, $d R_{\mathrm{T}}(r=0.61, p=0.026$, twotailed $t$ test; Fig. $3 D) . d R_{\mathrm{T}}$ was also positively correlated with the mean repetition length measured in the normal condition $(r=$ $0.77, p=0.001)$. In other words, syllables with long and variable repetition bouts tended to be affected by HVC temperature, whereas those with short bouts of more fixed lengths tended to be unaffected.

The two types could be predicted from the variability alone (linear classifier, error rate 0.077 ). We therefore used a threshold on the variability to classify repeating syllables into these two types in subsequent analyses (see Materials and Methods). The cooling effect on type I syllables was significant on aggregation $\left(n=8\right.$ syllables from 5 birds, slope $=0.4 \pm 0.1, p=1.3 \times 10^{-4}$, LMM; Fig. $3 C$ ), whereas the effect on type II syllables was not $(n=5$ from 3 birds, slope $=0.019 \pm 0.008, p=0.026$, LMM; Fig. $3 C)$. The difference in effects was significant between the two types $\left(p=2.7 \times 10^{-7}, \mathrm{LMM}\right)$.

A simple explanation for the shortening of syllable repetitions during cooling is that the song tempo is slowed while the dura- 
A

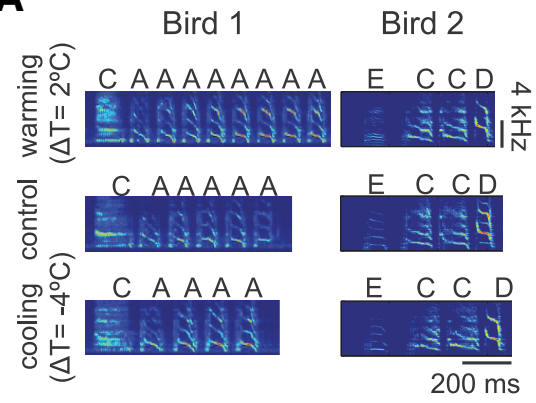

B
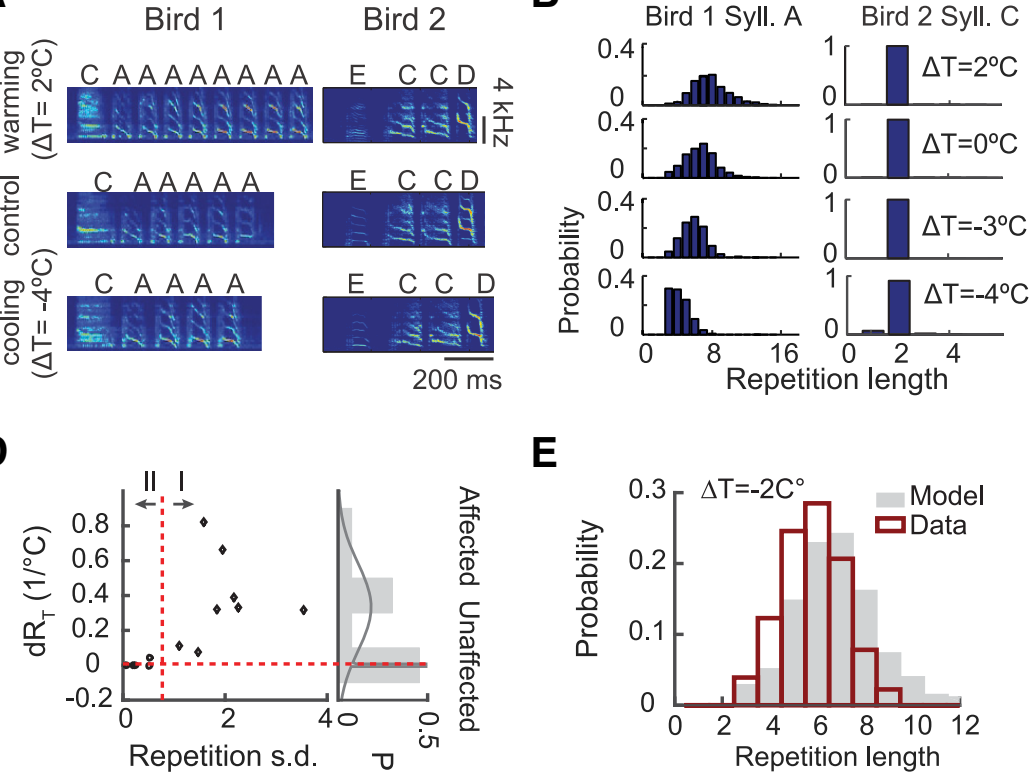

$E$

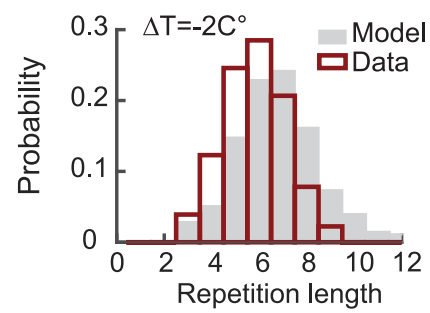

C

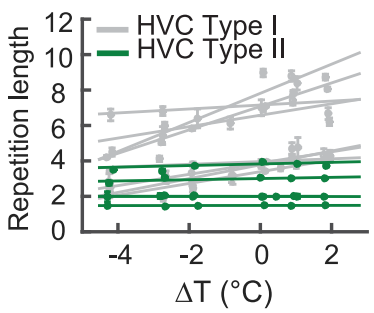

$\mathbf{F}$

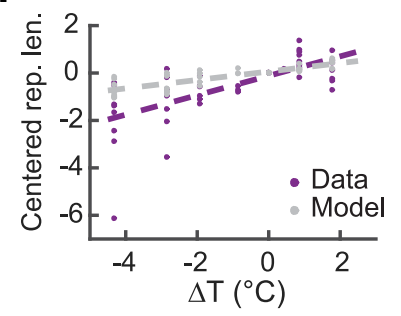

Figure 3. Effects of HVC temperature on syllable repetitions. $\boldsymbol{A}$, Cooling HVC reduces the length of some syllable repetitions. The first example shows a syllable repetition that has been affected by cooling and the second example shows a syllable repetition that has not been affected. Syllable " $\mathrm{A}$ " from Bird 1 repeats a variable number of times. The repetition length is shorter when temperature is lower and longer when temperature is higher. Syllable " $C$ " from Bird 2 only repeats twice and does not show such an effect. Representative repeat segments at different HVC temperatures are shown. The segments are aligned to the onset of the first syllable. B, Left, Histograms of repetition lengths of syllable " $A$ " at different HVC temperatures. Cooling systematically shifts the distribution, decreasing the mean number of repetitions. Right, Histograms of repetition lengths of syllable " $C$." Temperature change does not shift the distribution. $\boldsymbol{C}$, Mean repetition length of type I (gray lines) and type II (green lines) syllables at different temperatures. Temperature has a stronger effect on the type I syllables than the type II syllables ( $p=2.7 \times 10^{-7}$, LMM). $D$, Classification of type I and type Il syllables based on the SD of repetition length. Each point is a repeated syllable. The size of the temperature effect on repetition is given by $d R_{T}$, the slope estimated from the linear regression shown in C. Right, Bimodal distribution of the slopes fit with a mixture of two Gaussian distributions. The horizontal dashed line indicates the separation of the two Gaussian distributions. The vertical dashed line indicates the separation of the two categories by SD of repetition length distributions. To the right of the vertical line is the region for type I syllables and to the left is the region for type II syllables. $\boldsymbol{E}$, Repetition length distributions for an example syllable, both observed (gray) and predicted from a time conservation model (red). Compared with the distribution derived from the time conservation model, the observed effect of cooling HVC by $2^{\circ} \mathrm{C}$ shows a greater reduction of repetition length $\left(p=2.6 \times 10^{-11}, t\right.$ test, $N_{\text {model }}=1158$, $\left.N_{\text {observation }}=179\right)$. $\boldsymbol{F}$, Mean repetition lengths of the type I syllables as a function of temperature and the corresponding predictions from the time conservation model. The model-generated effect of HVC temperature on repetition length is significantly weaker than that observed in the real data $\left(p=8 \times 10^{-4}\right.$, ANCOVA).

tions of repetition bouts, which could be encoded in a different brain area, are unaffected by the cooling. In this scenario, fewer syllables can "fit" in a repeat bout because the syllables and gaps are longer. We compared this theoretical prediction to the actual data. One example is shown in Figure $3 E$ (syllable "A" from Bird 1 at $\Delta T=-2^{\circ} \mathrm{C}$ ). The predicted reduction in mean repetition length was significantly smaller than that observed $(p=2.6 \times$ $10^{-11}$, two-tailed $t$ test). This was true across the population of type I syllables (Fig. $3 F$ ). Compared with the predicted values, the observed data showed significantly larger shifts in mean repetition lengths as HVC temperature was changed ( $p=8 \times 10^{-4}$, ANCOVA). Therefore, the cooling-induced reduction in syllable repetition is not simply a byproduct of slowed song tempo.

\section{Branch points}

A branch point in the song syntax consists of probabilistic transitions from one syllable to multiple syllables. Two examples are shown in Figure 4A. In the first example, syllable "K" can transition to syllable " $\mathrm{B}$ " with a probability of 0.68 or to syllable " $\mathrm{D}$ " with a probability of 0.32 (Bird 3). In the second example, syllable "F" can be followed by syllable "B" with a probability of 0.60 , syllable "G" with a probability of 0.36 , or syllable "J" with a small probability of 0.04 (Bird 7). We identified a total of 12 branch points in the songs of five birds. The branch points were defined after controlling for syllable misclassification. In the normal condition, all transition probabilities were stable across days $(p>$ 0.05 , Fisher's exact test).
Cooling HVC affected transition probabilities at branch points. In the first example shown in Figure $4 A$, when HVC was cooled by $4^{\circ} \mathrm{C}$, the probability for the transition "KB" was reduced to 0.45 , whereas the probability for "KD" was enhanced to 0.55 . In the second example, the transition probability of "FJ," which was very small in the normal condition, was enhanced to 0.24 . These changes were highly significant $\left(p=1.7 \times 10^{-5}\right.$ and $p=1.1 \times 10^{-6}$, respectively, Fisher's exact test). Transition probabilities for 9 of 12 branch points were significantly dependent on temperature $\left(p<0.05, \chi^{2}\right.$ test of independence or Fisher's exact test for counts $<10$ ).

Cooling HVC also affected the variability of syllable sequences. The randomness of syllable transitions at a branch point can be quantified by transition entropy (Okanoya and Yamaguchi, 1997; Woolley and Rubel, 1997; Sakata and Brainard, 2009). A deterministic transition has the lowest entropy and the value is 0 . The most random transition, in which the transitions to all $N$ targets are equally probable, has the maximum entropy, with a value of $\log _{2} N$. We calculated transition entropy for the 12 branch points. Cooling HVC significantly increased the transition entropy of four branch points ( $p=0.010,0.017,0.014,0.023$, two-tailed $t$ test on the slope of transition entropy vs temperature), slightly reduced the transition entropy of one ( $p=0.025$, two-tailed $t$ test on slope), and had no significant effect on the rest (Fig. 4B). The extent of the cooling effect on sequence variability depended on the transition entropy of the branch points under normal conditions. The branch points with 
A
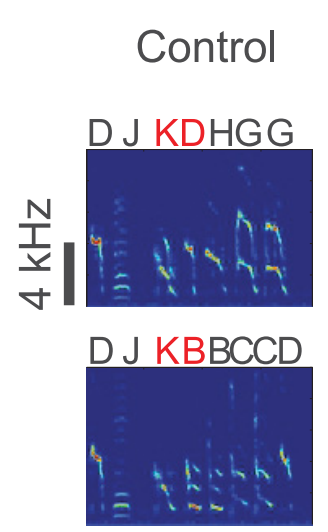

$200 \mathrm{~ms}$
Cooling

$\left(\triangle \mathrm{T}=-4^{\circ} \mathrm{C}\right)$
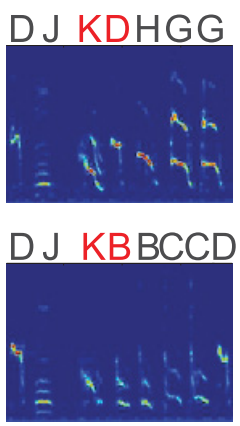

Control

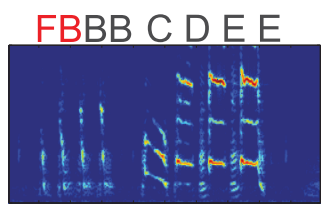

FGGGGG GGGG

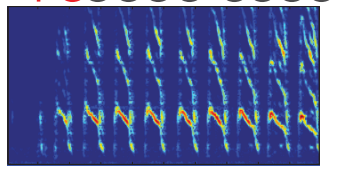

FJJJJJJJJJJJJ H

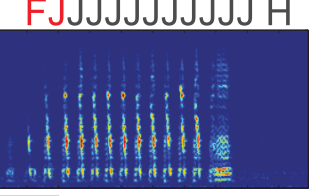

$200 \mathrm{~ms}$

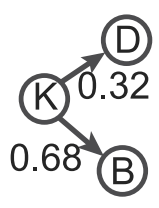

$\mathrm{n}=201$

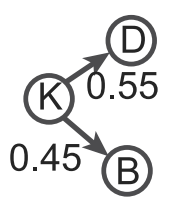

$\mathrm{n}=162$

B

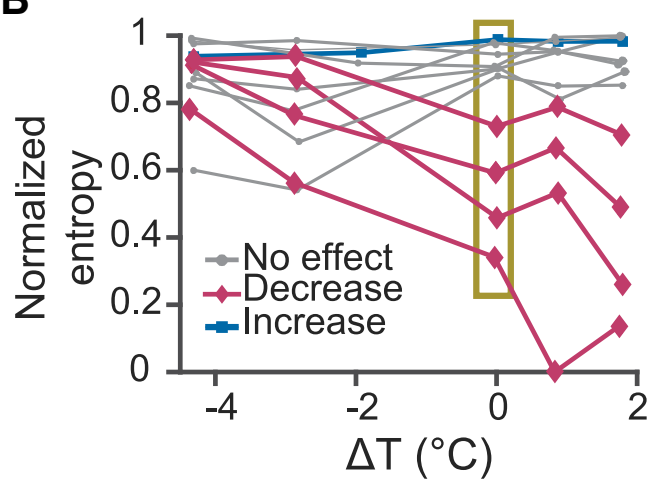

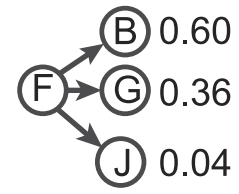

$\mathrm{n}=152$
Cooling

$\left(\triangle T=-4^{\circ} \mathrm{C}\right)$
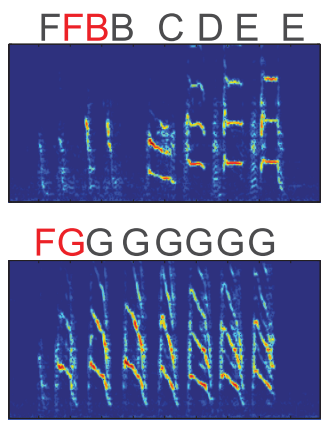

FJJJJJUJJH
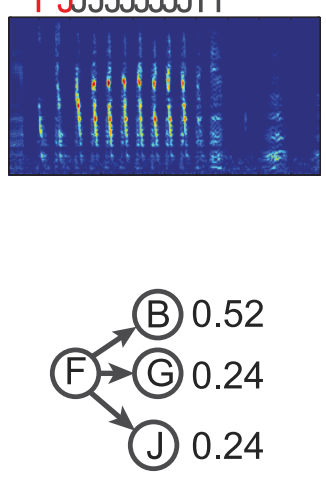

$\mathrm{n}=148$

\section{C}

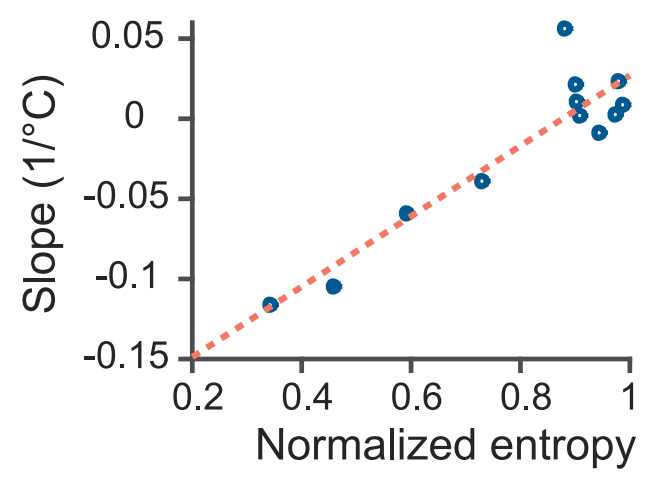

Figure 4. Effects of HVC temperature on branch points in the song syntax. $A$, Two examples of branch points affected by HVC temperature. Left, Top, Spectrograms of song segments showing that syllable " $K$ " can be followed by either syllable " $B$ " or syllable "D." Bottom, Transition probabilities from " $K$ " to " $B$ " or " $D$ " under control and $4^{\circ} \mathrm{C}$ cooling conditions. Right, Top, Spectrograms showing that syllable " $F$ " can be followed by short repeat "B," long repeat "G," or "J." Bottom, Transition probabilities from " $F$ " to "B," "G," or "J" under the normal and $4{ }^{\circ} \mathrm{C}$ cooling conditions. $B$, Dependence of the transition entropy on HVC temperature for all branch points. Entropies are normalized by their maximum possible values. Magenta curves are branch points that show a significant decrease in transition entropy as temperature increases; blue curves show a significant increase; gray curves show branch points with no significant change ( $t$ test on slope). Transition entropies at $\Delta T=0$ are within the box and are used on the abscissa in $C$. C, Slopes of the normalized entropy versus $\Delta T$ curve plotted with respect to the normalized entropy at $\Delta T=0$. The correlation is significant $(r=$ $0.92, p=1.2 \times 10^{-5}, t$ test).

lower transition entropy at normal temperature tended to increase their transition entropy more when HVC was cooled $(r=0.92, p=$ $1.2 \times 10^{-5}$, $t$ test; Fig. $4 C$ ). At the same time, all of the branch points that showed either no change or a slight increase in entropy had baseline values of entropy that were close to the maximum possible value to begin with. That is, cooling HVC increased the randomness of the syllable transitions that were closer to being stereotypical under normal conditions, but did not strongly affect the transitions that were already highly random.

A more drastic change in syllable sequence would be the appearance of novel transitions. However, across the total of 286 possible transition types inspected ( $n=5$ birds), we observed only two instances of novel transitions in one bird (Bird 7) at the lowest temperature conditions. Therefore, cooling primarily affected the probabilities of the existing transitions instead of creating new transitions.

Song bouts

HVC temperature also affected the lengths of song bouts. We defined the end of a song bout as a syllable followed by either a silent period of $>2$ s or introductory notes. Excluding introductory notes, a song bout consisted of $\sim 12-21$ syllables and lasted 
A
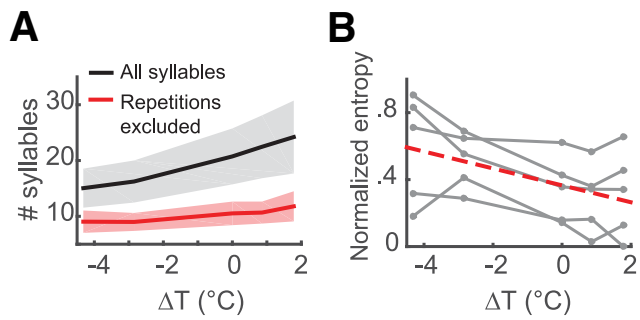

C

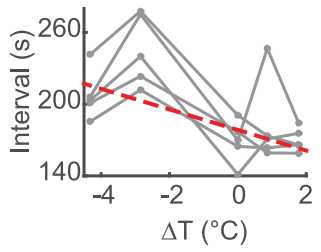

Figure 5. Effects of HVC temperature on song bouts. $A$, Number of syllables within a song bout with respect to changes in HVC temperature. Shaded area is SEM The black trace uses the full number of syllables; the red trace excludes syllable repetitions. Both trends are significantly positive in aggregation. $\boldsymbol{B}$, Normalized transition entropy of the ending syllables versus HVC temperature change. Each gray line is from one bird. Red dashed line is the fitted linear model of the population. The slope is significantly smaller than $0\left(p=6.6 \times 10^{-6}, \mathrm{LMM}\right)$. C, Intersong intervals versus HVC temperature change. Each gray line is from one bird. Red dashed line is the fitted linear model of the population. The slope is mildly negative ( $p=0.04, \mathrm{LMM})$.

for $\sim 1.4-2.4 \mathrm{~s}$ (25th-75th percentile, $n=5$ birds). The number of syllables within a bout was affected by temperature changes in HVC $\left(p=4.0 \times 10^{-46}\right.$, LMM $)$, decreasing $\sim 0.9$ syllables per degree Celsius decrease in temperature (Fig. $5 A$, black curve). This effect could not be fully explained by variations in syllable repetition length. When counting repeated syllables only once, the dependence of song bout length on HVC temperature still existed ( $p=4.6 \times 10^{-25}$, LMM), with a shortening of $\sim 0.3$ syllables per bout for every degree Celsius temperature drop in HVC (Fig. 5A, red curve). A possible explanation is that cooling HVC might increase the probability for a song to stop at syllables after which the song rarely ends in the normal condition. If so, then the entropy of the probability distribution of the ending syllables should increase with cooling HVC; this was indeed the case $\left(p=6.6 \times 10^{-6}\right.$, LMM; Fig. $\left.5 B\right)$. Warming HVC had the opposite effect (Fig. $5 B$ ). In the normal condition, birds spontaneously produced a song approximately every 20-102 s (25th75th percentile). Temperature manipulation in HVC slightly changed the frequency of song production, with the mean interval between songs increasing by $9 \mathrm{~s}$ for every degree Celsius decrease of temperature ( $p=0.04, \mathrm{LMM})$. Overall, the number of vocal outputs within a given time period is positively correlated with HVC temperature.

\section{Cooling RA has minimal effects on the song syntax}

To test whether the effects on song syntax were due to modification of the neural activity in downstream areas, we manipulated the temperature of RA. RA is a major motor area for birdsong production and is directly innervated by HVC (Nottebohm et al., 1976). Because RA is located deep ( $2 \mathrm{~mm}$ ) below the brain surface, we used thermally conductive probes to manipulate its temperature (Long and Fee, 2008).

Cooling RA produced only mild effects on the durations of the syllables and gaps. Cooling RA stretched the syllables by $0.9 \pm$ $0.1 \% /{ }^{\circ} \mathrm{C}(n=22$ in 2 birds; Fig. $6 A)$ and the gaps by $0.5 \pm$ $0.3 \% /{ }^{\circ} \mathrm{C}(n=34$ in 2 birds; Fig. $6 B)$. According to a prior study, RA cooling leads to cooling in HVC with the change of temperature $\sim 30 \%$ of that in RA (Long and Fee, 2008). The effect of RA cooling on syllable durations could be explained by the collateral temperature change in HVC ( $p=0.94, t$ test). However, the effect of cooling RA on gap durations is slightly less than can be accounted for by the collateral temperature changes in $\operatorname{HVC}(p=$ $0.01, t=$ test $)$. In the songs of the two birds in which we manipulated RA temperature, there were four type I repeated syllables (Fig. 6E) and seven branch points. Figure 6, $C$ and $D$, shows an example of a type I syllable for which the distribution of repetition lengths was not shifted by changes in RA temperature. For all four type I repeated syllables, changes in mean repetition length were not significantly dependent on RA temperature ( $p=0.27, \mathrm{LMM})$ and were significantly smaller than those observed in HVC cooling $\left(p=8.2 \times 10^{-7}\right.$, LMM; Fig. $\left.6 F\right)$. At branch points, no significant trends in the changes of transition entropy were observed as RA was cooled ( $p>0.05, t$ test; Fig. $6 G$ ) regardless of the transition entropies in the normal condition (Fig. $6 H$ ). Finally, changes in RA temperature did not affect the length of song bouts ( $p=$ 0.60 , LMM) or the rate of song production as measured by intersong intervals $(p=0.39, \mathrm{LMM})$. In conclusion, RA temperature manipulation showed minimal impact on the song syntax.

\section{Hemispherical asymmetry}

To evaluate the relative contributions of the left and right HVCs to cooling effects on Bengalese finch song, we performed unilateral cooling. We found that the stretching of syllable durations was mainly due to left HVC cooling ( 37 syllables in 5 birds, $p=$ $1.2 \times 10^{-8}$, paired $t$ test; Fig. $7 A$ ). The mean stretch from cooling the left HVC was $2.0 \pm 0.9 \% /{ }^{\circ} \mathrm{C}\left(p=4.7 \times 10^{-15}, t\right.$ test for difference from 0 ); the mean stretch from cooling the right $\mathrm{HVC}$ was $0.3 \pm 1.0 \% /{ }^{\circ} \mathrm{C}(p=0.09, t$ test for difference from 0$)$. There was no correlation between the changes in syllable durations observed from cooling the left HVC and from cooling the right $\operatorname{HVC}(r=0.008, p>0.05, t$ test; Fig. $7 B)$.

Cooling either hemisphere stretched the intersyllable gaps by approximately the same amount (left: $2.1 \pm 2.6 \% /{ }^{\circ} \mathrm{C}$; right: $2.6 \pm$ $3.6 \% /{ }^{\circ} \mathrm{C}, p=0.35$, paired $t$ test; Fig. $7 C$ ). There was an anticorrelation between the stretches of gaps from left and right $\mathrm{HVC}$ cooling $\left(r=-0.31, p=8 \times 10^{-3}\right.$, shuffle test; Fig. $\left.7 D\right)$.

We found that some of the type I syllable repetitions were primarily affected by cooling only one of the hemispheres, whereas others were affected by cooling either hemisphere. To assess hemispheric lateralization, we compared the effects of temperature change on syllable repetition under unilateral cooling and bilateral cooling in four birds. Figure $8, A-C$, shows three examples of the different ways in which repetition length was affected by unilateral/bilateral cooling. Figure $8 \mathrm{~A}$ shows a case in which cooling the right HVC produced similar effects on syllable repetitions as bilateral cooling, whereas the effect from cooling the left HVC was much smaller than the other two conditions $\left(F=21.1, p=4.1 \times 10^{-4}\right.$, ANCOVA $)$. In contrast, Figure $8 B$ shows an example in which the effect of cooling the left but not the right HVC was close to bilateral cooling $(F=10.5, p=4.4 \times$ $10^{-3}$, ANCOVA). For some cases, cooling either HVC produced similar effects as bilateral cooling $(F=1.4, p=0.29)$, as shown in Figure $8 C$. Among the 10 repeated syllables, three were significantly affected by cooling the left HVC but not the right, three were affected by the right but not the left, three were affected by both hemispheres, and one was not affected by either hemisphere (two-tailed $t$ test on slopes of repetition length vs temperature; Fig. $8 D$ ). To evaluate the strength of lateralization, for each syllable, we selected the hemisphere where the cooling effect was stronger (the side with greater slope) and compared that effect with the bilateral cooling effect. We found that dominant side cooling accounts for $\sim 70 \%$ of the effect on syllable repetition 
A

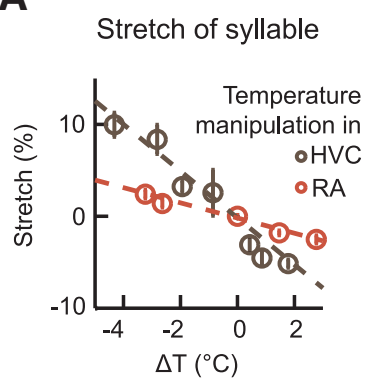

B

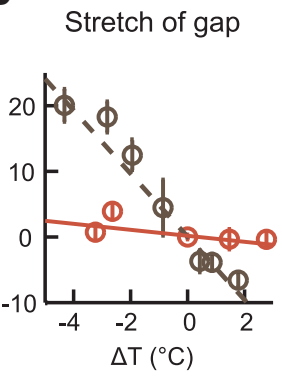

C
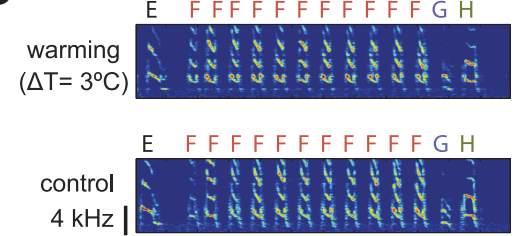

\begin{tabular}{c} 
E FFFFFFFFFFF GH \\
cooling \\
$\left(\Delta \mathrm{T}=-3^{\circ} \mathrm{C}\right)$ \\
\cline { 2 - 2 }
\end{tabular}

$200 \mathrm{~ms}$
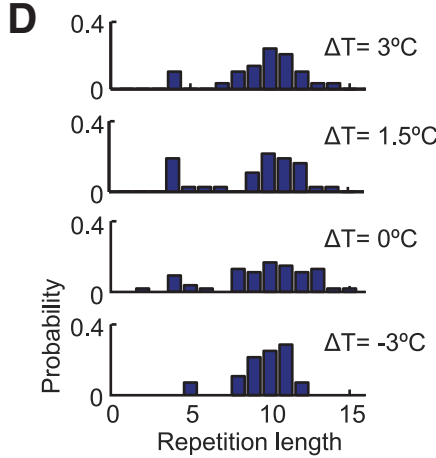

H
E

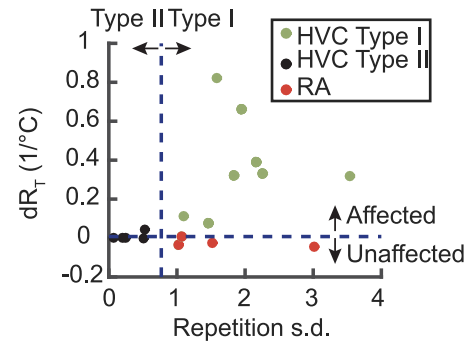

F

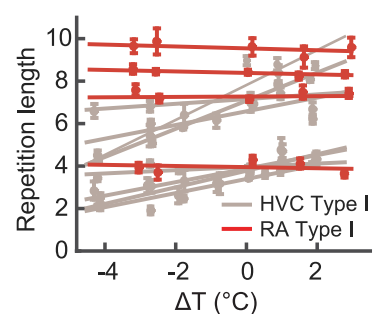

G

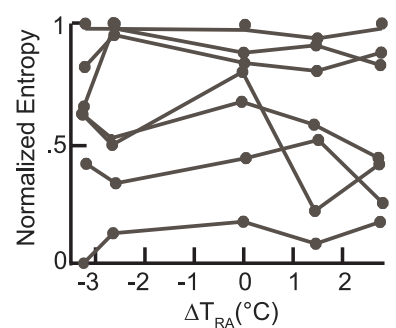

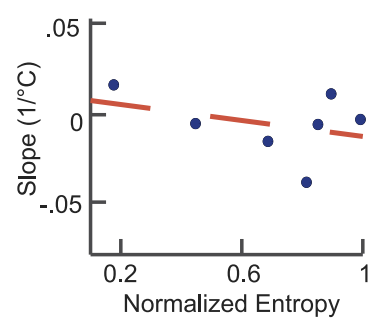

Figure 6. Effects of RA temperature on song timing and syntax. A, Mean fractional stretch of syllables $(n=22)$ at various RA temperatures compared with the stretch with changing the temperature of HVC. Error bars indicate SEM. B, Mean fractional stretch of gaps $(n=34)$ at various RA temperatures compared with the stretch with changing the temperature of HVC. C, Spectrograms showing the repetition of syllable "F" at different RA temperatures. D, Histograms of the repetition length of syllable "F" under different RA temperatures. Cooling RA does not shift the distribution of the repetition length. $\boldsymbol{E}$, RA cooling does not decrease syllable repetitions for type I repeated syllables as defined with the variability of repetition length at the normal conditions (red dots). This is compared with the type I (purple) and type II (black) syllables from the HVC cooling experiments. $\boldsymbol{F}$, Mean repetition length of the type I repeated syllables at different temperatures under RA cooling (red) compared with HVC cooling (gray). Error bars indicate SEM. G, Normalized transition entropy of the seven branch points from two birds at different RA temperatures. All curves have slopes that are not significantly different from zero. $\boldsymbol{H}$, Slope of the curves with respect to the normalized entropy at $\Delta T=0$. The slopes are not correlated with the transition entropy at $\Delta T=0(p=0.21, t$ test $)$.

(slope from dominant side cooling divided by slope from bilateral cooling $=$ 0.67; Fig. 8E).

Unilateral cooling also affected the transition probabilities at the branch points. For the four branch points that showed a significant increase in entropy under bilateral cooling (Fig. 4), cooling of at least one hemisphere significantly increased the transition entropy by itself (Fig. $8 F-I$ ). The branch points that were not significantly affected by bilateral cooling remained unaffected. For three of the affected branch points, there were no significant differences among left, right, or bilateral HVC cooling (Fig. 8F,G,I; ANCOVA, $p>0.05$ ). For the remaining branch point, right cooling had a significantly weaker effect than bilateral cooling $(F=5.87, p=0.0184$, ANCOVA $)$; the effect of left cooling was statistically indistinguishable from the effect from bilateral cooling, although the average was lower (Fig. $8 \mathrm{H}$ ).

Cooling HVC in either hemisphere reduced song bout length in 4 of 5 birds $(p<0.05$, LMM); for the remaining bird, only left HVC cooling produced an effect. This effect persisted after syllable repetitions were taken out. Within the same subjects, the hemispheric dominance in
A
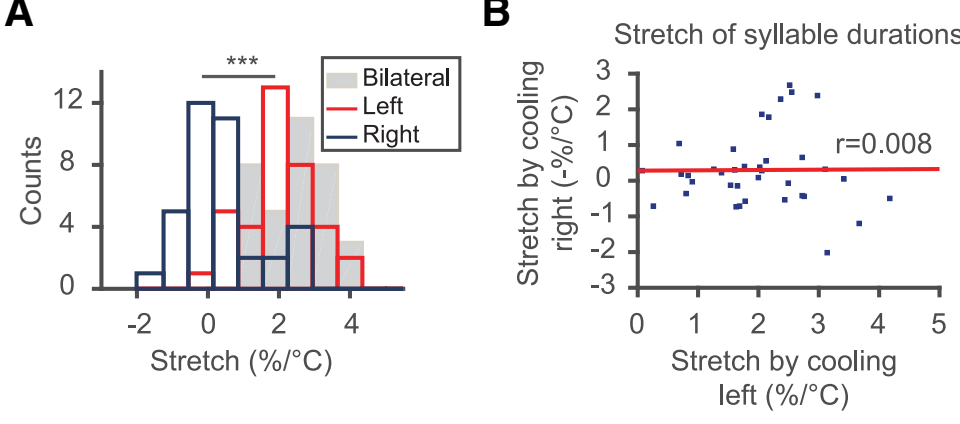

C

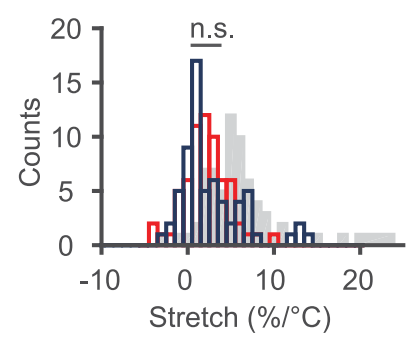

D

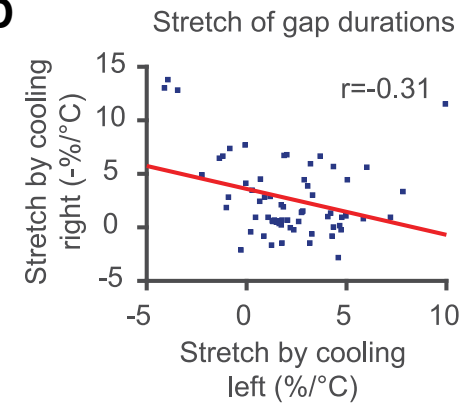

Figure 7. Effects of unilateral HVC temperature manipulation on syllable and gap durations. $\boldsymbol{A}$, Histograms of syllable stretch under bilateral (gray), left (red), and right (blue) HVC cooling. Cooling the left HVC shows greater effect on syllable durations than cooling the right HVC ( $p=1.2 \times 10^{-8}$, paired $t$ test). $\boldsymbol{B}$, Correlation between syllable stretch with cooling the left and the right HVCs. Effects from the two hemispheres are not correlated $(r=0.008)$. C, Histograms of gap stretch under bilateral (gray), left (red), and right (blue) HVC cooling. The two hemispheres show similar degrees of effect on gap timing ( $p=0.35$, paired $t$ test). D, Gap stretch by cooling the left and right HVC are anticorrelated $(r=-0.31)$. 
A

B
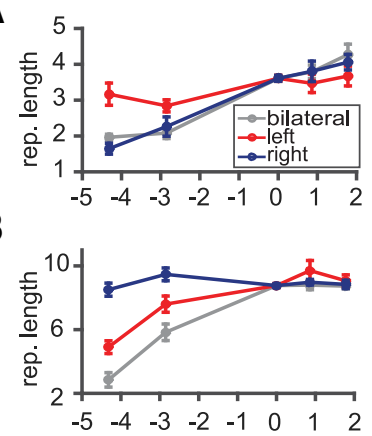

C

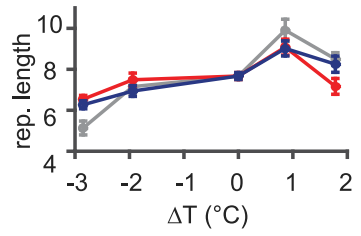

D

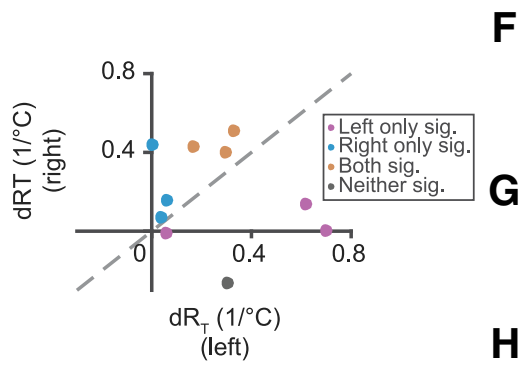

E

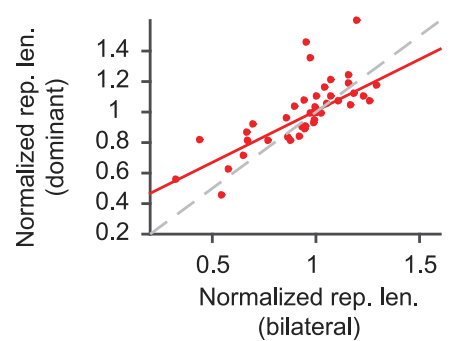

F
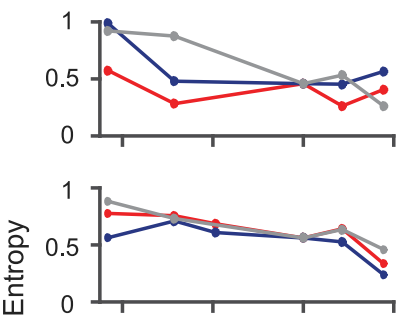

H

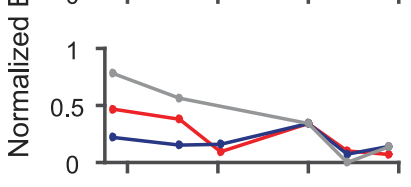

I

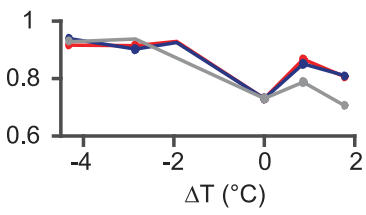

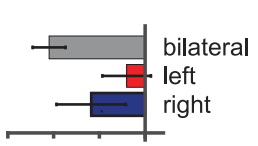
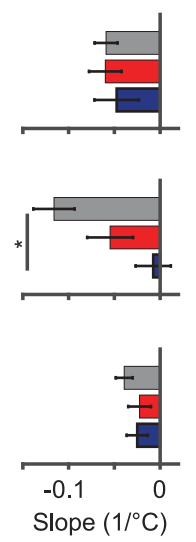

Figure 8. Effects of unilateral HVC temperature manipulation on song syntax. A-C, Examples of variation in mean repetition length with respect to HVC temperature. Effects of left HVC cooling (red lines), right HVC cooling (blue lines), and bilateral cooling (gray lines) are shown. In A, right HVC cooling has a similar effect to bilateral cooling ( $p=0.99$, Tukey-Kramer post hoc test), whereas left cooling has a smaller effect than bilateral cooling $\left(p=9.2 \times 10^{-4}\right)$; in $\boldsymbol{B}$, left cooling is similar to bilateral cooling $(p=0.39)$, whereas right cooling has no effect; in $C$, both left and right cooling

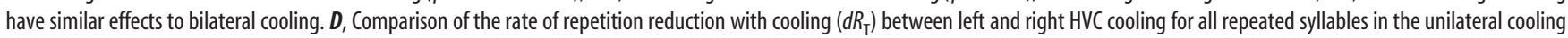
experiment. The gray line is $y=x . \boldsymbol{E}$, Comparison of mean repetition length between dominant side cooling and bilateral cooling. The repetition lengths are normalized to the mean length in the normal condition. Red line is the linear fit. The gray line is $y=x . \boldsymbol{F}-\boldsymbol{I}$, Changes of transition entropy with unilateral and bilateral HVC cooling for the four significantly affected branch points (Fig. 4). Entropies are normalized by the maximum possible values. Syllables in $\boldsymbol{F}, \boldsymbol{G}$, and $\boldsymbol{I}$ show no difference among the three conditions (ANCOVA, $p>0.05$ ); the syllable in $\boldsymbol{H}$ shows a significantly weaker effect for right HVC cooling than bilateral cooling ( $F=5.87, p=0.0184$, ANCOVA).

the control of song bout length was consistent with that in the syllable repetitions and in the branch points. No obvious change was observed in the rate of song production with unilateral cooling except in one bird.

\section{Discussion}

Variability in syllable sequences is a prominent feature of Bengalese finch song. From rendition to rendition, syllables can repeat a variable number of times and different syllables can be chosen at branch points. Syllable sequencing thus requires making choices among multiple possibilities. Using a cooling technique, we have shown that HVC plays a key role in making such choices. Cooling HVC reduced repetition lengths of long and variably repeated syllables and increased the randomness of syllable sequences. These results suggest that HVC is a prime candidate for encoding song syntax. Previous experiments in the zebra finch established HVC's role in controlling the timing of features at the syllable level (Hahnloser et al., 2002; Fee et al., 2004; Long and Fee, 2008; Long et al., 2010; Lynch et al., 2016; Picardo et al., 2016). Our experiments suggest that HVC plays an additional role of determining probabilistic transitions between syllables. This is inconsistent with the idea of strict hierarchical control of birdsong, in which song syntax is at a level higher than that of syllables and is determined in an area upstream of HVC, perhaps NIf (Hosino and Okanoya, 2000) or Uva (Williams and Vicario, 1993). Instead, HVC is critical for both controlling syllables and encoding song syntax.

Although the cooling technique has been used before on the zebra finch (Long and Fee, 2008; Aronov and Fee, 2011; Hamaguchi et al., 2016) and the canary (Goldin et al., 2013), our experiments provide novel insights into HVC's role in encoding probabilistic syllable transitions, a crucial aspect of song syntax in many species. Zebra finch songs typically have rigid sequences
(Sossinka and Böhner, 1980), making this species unsuitable for studying variability in syllable sequences. Songs of the canary have elaborate syntax with syllable repetitions and probabilistic transitions (Gardner et al., 2005; Markowitz et al., 2013). However, the study that cooled HVC in the canary was focused on the interaction between HVC inputs and the nonlinear dynamics of respiratory control (Goldin et al., 2013) and did not investigate the song syntax. It would be interesting to repeat the cooling experiment on the canary and determine whether our findings on the song syntax extend to other species with variable syllable sequences.

HVC projects directly to RA and Area X, the input station of the anterior forebrain pathway (AFP; Nottebohm et al., 1976). We showed that cooling RA, which affects RA's intrinsic dynamics as well as the inputs to RA from other areas, has a minimal effect on the song syntax, ruling out RA in determining the song syntax. Song syntax is not encoded in AFP either because lesioning the lateral magnocellular nucleus of the anterior nidopallium, the main output station of AFP, does not affect song syntax in the Bengalese finch (Hampton et al., 2009). Therefore, the effects of HVC cooling on song syntax are not due to altered inputs to RA or Area X.

Singing in songbirds is controlled bilaterally. The HVCs in the two hemispheres control sound production in ipsilateral halves of the syrinx (Goller and Suthers, 1996). Our unilateral cooling results showed that syllable durations were mostly affected by cooling left HVC, suggesting that left HVC controls feature timings in syllables. In contrast, both sides affected gap durations, with the amount of stretching anticorrelated for left and right cooling. These results suggest that syllables are mainly encoded in left HVC, whereas gaps are influenced by both HVCs. The left dominance of syllable control in the Bengalese finch is consistent 
with a previous experiment showing that devocalization of the left but not the right syrinx destroyed the majority of Bengalese finch song syllables (Secora et al., 2012).

In the zebra finch, the left and right HVCs alternate in controlling the song tempo (Long and Fee, 2008; Wang et al., 2008). This difference in hemispherical dominance between the zebra finch and the Bengalese finch may be due to the difference in the song variability.

Similarly to the gaps, syllable transition probabilities were affected by cooling both HVCs. For long and variable repetitions, some were mainly affected by only left or right HVC cooling, some by both. Randomization of sequences at branch points was seen in cooling both sides, although in some cases one side dominated. Given that syllables are encoded in left HVC, it is easy to understand how left HVC cooling could affect transition probabilities by affecting the synapses and neurons encoding the syllables. It is harder to explain how right HVC cooling can contribute to, and in some cases even dominate, changes in transition probabilities. One possibility is that the right HVC contributes to the initiation and termination dynamics of inspiration in the brainstem during gaps (Andalman et al., 2011) and influences left HVC activity through the brainstem signals triggered by inspiration and delivered bilaterally to HVC via Uva (Schmidt, 2003; Andalman et al., 2011). This is consistent with our observation that right HVC can control gap durations. In this view, syllable transitions involve the feedback loop connecting the left and right HVCs through the brainstem and Uva (Andalman et al., 2011).

Our results are consistent with the model that probabilistic syllable selections are determined within HVC (Chang and Jin, 2009; Jin, 2009; Hanuschkin et al., 2011; Wittenbach et al., 2015). A crucial aspect of the model is that a winner-take-all competition between briefly coactive syllable chains takes place at the transition points (Chang and Jin, 2009; Jin, 2009). The competition is mediated through the inhibitory interneurons in HVC (Mooney and Prather, 2005; Kosche et al., 2015), which necessarily requires that the syllable chains reside in the same hemisphere (Jin, 2009). This agrees with our observation that syllables are encoded in left HVC. The model allows external inputs from Uva or NIf to influence transition probabilities by biasing the excitabilities of syllable chains. In particular, the model distinguishes type I and type II syllable repetitions (Wittenbach et al., 2015). Analysis of long and variable repeated syllables (type I) showed that these syllables should be initially sustained by strong auditory feedback from NIf to the repeating chain such that initial repeat probability is close to 1 . As the syllable repeats, the auditory feedback weakens due to synaptic adaptation, leading to termination of the repetition. For short repeats with minimal variability (type II), such strong auditory feedback is unnecessary. Indeed, deafening Bengalese finches reduced repetition lengths of type I repeated syllables while leaving type II repeated syllables intact (Wittenbach et al., 2015). It is possible that cooling HVC weakens the NIf synapses in HVC, reducing the repetition lengths of type I but not type II syllables. Similar reasoning can be applied to the branch points. We observed that cooling HVC tends to equalize the transition probabilities at branch points and thus increases the randomness of the syllable sequences. It is possible that auditory feedback enhances transition probability to a particular syllable by specifically targeting the neurons that encode it (Sakata and Brainard, 2006; Hanuschkin et al., 2011; Wittenbach et al., 2015). Cooling the NIf synapses in HVC reduces such bias, revealing more random transitions within HVC's intrinsic network promoted by the Uva inputs at each syllable transition. More detailed analysis of the model's responses to cooling should reveal modifications needed for the model to fully explain our cooling results.

Our experiments do not rule out the possibility that song syntax is generated within the feedback loop involving HVC, RA, the brainstem, and Uva (Schmidt, 2003; Ashmore et al., 2005). Perhaps other areas in the loop in addition to HVC also play a role in determining syllable sequences. RA can be excluded because cooling RA has a minimal effect on the syntax. A recent experiment cooled Uva in the zebra finch and showed that the song tempo slowed (Hamaguchi et al., 2016). Cooling Uva in the Bengalese finch could be useful to address its role on syntax. However, one needs to be cautious in analyzing such experiments because it could be that Uva affects the song indirectly by modulating HVC dynamics.

One caveat is that surrounding areas are also inevitably affected when cooling HVC. Based on the relationship between distance and temperature drop (Aronov and Fee, 2011), we estimate that cooling HVC or RA should produce similar temperature changes in NIf. Because cooling RA had minimal effects on syntax, we can rule out the possibility that the effects on song syntax are due to the collateral temperature change in NIf. Uva is relatively distant from HVC, so temperature change in Uva from manipulating temperature in $\mathrm{HVC}$ is negligible (Hamaguchi et al., 2016). Other areas, such as the auditory area field $\mathrm{L}$ and the avalanche nucleus, an area mutually connecting to NIf and HVC (Akutagawa and Konishi, 2010), could be cooled at most by $\sim 2^{\circ} \mathrm{C}$. Further investigation is needed to determine whether this is enough to change the song syntax.

In summary, we find that HVC is a key site for encoding song syntax in the Bengalese finch. Syllable durations are mostly controlled by left HVC, whereas the gap durations and the song syntax can be influenced bilaterally.

\section{References}

Akutagawa E, Konishi M (2010) New brain pathways found in the vocal control system of a songbird. J Comp Neurol 518:3086-3100. CrossRef Medline

Andalman AS, Foerster JN, Fee MS (2011) Control of vocal and respiratory patterns in birdsong: dissection of forebrain and brainstem mechanisms using temperature. PLoS One 6:e25461. CrossRef Medline

Aronov D, Fee MS (2011) Analyzing the dynamics of brain circuits with temperature: design and implementation of a miniature thermoelectric device. J Neurosci Methods 197:32-47. CrossRef Medline

Ashmore RC, Wild JM, Schmidt MF (2005) Brainstem and forebrain contributions to the generation of learned motor behaviors for song. J Neurosci 25:8543-8554. CrossRef Medline

Chang W, Jin DZ (2009) Spike propagation in driven chain networks with dominant global inhibition. Phys Rev E Stat Nonlin Soft Matter Phys 79:051917. CrossRef Medline

Chomsky N (1965) Aspects of the theory of syntax. Cambridge, MA: MIT.

Cromwell HC, Berridge KC (1996) Implementation of action sequences by a neostriatal site: a lesion mapping study of grooming syntax. J Neurosci 16:3444-3458. Medline

Doupe AJ, Kuhl PK (1999) Birdsong and human speech: common themes and mechanisms. Annu Rev Neurosci 22:567-631. CrossRef Medline

Fee MS, Kozhevnikov AA, Hahnloser RH (2004) Neural mechanisms of vocal sequence generation in the songbird. Ann N Y Acad Sci 1016:153-170. CrossRef Medline

Fisher RA (1922) On the interpretation of $\chi 2$ from contingency tables, and the calculation of P. Journal of the Royal Statistical Society 85:87-94.

Gardner TJ, Naef F, Nottebohm F (2005) Freedom and rules: the acquisition and reprogramming of a bird's learned song. Science 308:1046-1049. CrossRef Medline

Gelman A, Hill J (2006) Data analysis using regression and multilevel/hierarchical models. Cambridge: Cambridge University.

Gibb L, Gentner TQ, Abarbanel HD (2009) Brain stem feedback in a computational model of birdsong sequencing. J Neurophysiol 102:17631778. CrossRef Medline 
Goldin MA, Alonso LM, Alliende JA, Goller F, Mindlin GB (2013) Temperature induced syllable breaking unveils nonlinearly interacting timescales in birdsong motor pathway. PLoS One 8:e67814. CrossRef Medline

Goller F, Suthers RA (1996) Role of syringeal muscles in controlling the phonology of bird song. J Neurophysiol 76:287-300. Medline

Hahnloser RH, Kozhevnikov AA, Fee MS (2002) An ultra-sparse code underlies the generation of neural sequences in a songbird. Nature 419:6570. CrossRef Medline

Hamaguchi K, Tanaka M, Mooney R (2016) A distributed recurrent network contributes to temporally precise vocalizations. Neuron 91:680693. CrossRef Medline

Hampton CM, Sakata JT, Brainard MS (2009) An avian basal gangliaforebrain circuit contributes differentially to syllable versus sequence variability of adult Bengalese finch song. J Neurophysiol 101:3235-3245. CrossRef Medline

Hanuschkin A, Diesmann M, Morrison A (2011) A reafferent and feedforward model of song syntax generation in the Bengalese finch. J Comput Neurosci 31:509-532. CrossRef Medline

Hosino T, Okanoya K (2000) Lesion of a higher-order song nucleus disrupts phrase level complexity in Bengalese finches. Neuroreport 11:2091-2095. CrossRef Medline

Jin DZ (2009) Generating variable birdsong syllable sequences with branching chain networks in avian premotor nucleus HVC. Phys Rev E Stat Nonlin Soft Matter Phys 80:051902. CrossRef Medline

Jin DZ, Kozhevnikov AA (2011) A compact statistical model of the song syntax in Bengalese finch. PLoS Comput Biol 7:e1001108. CrossRef Medline

Kosche G, Vallentin D, Long MA (2015) Interplay of inhibition and excitation shapes a premotor neural sequence. J Neurosci 35:1217-1227. CrossRef Medline

Lashley KS (1951) The problem of serial order in behavior. In: Cerebral Mechanisms in Behavior. Indianapolis: Bobbs-Merrill.

Long MA, Fee MS (2008) Using temperature to analyse temporal dynamics in the songbird motor pathway. Nature 456:189-194. CrossRef Medline

Long MA, Jin DZ, Fee MS (2010) Support for a synaptic chain model of neuronal sequence generation. Nature 468:394-399. CrossRef Medline

Lynch GF, Okubo TS, Hanuschkin A, Hahnloser RH, Fee MS (2016) Rhythmic continuous-time coding in the songbird analog of vocal motor cortex. Neuron 90:877-892. CrossRef Medline

Markowitz JE, Ivie E, Kligler L, Gardner TJ (2013) Long-range order in canary song. PLoS Comput Biol 9:e1003052. CrossRef Medline

McLachlan G (2004) Discriminant analysis and statistical pattern recognition. New York: Wiley.

Mooney R, Prather JF (2005) The HVC microcircuit: the synaptic basis for interactions between song motor and vocal plasticity pathways. J Neurosci 25:1952-1964. CrossRef Medline

Newcombe RG (1998) Two-sided confidence intervals for the single proportion: comparison of seven methods. Stat Med 17:857-872. CrossRef Medline

Nottebohm F, Stokes TM, Leonard CM (1976) Central control of song in the canary, Serinus canarius. J Comp Neurol 165:457-486. CrossRef Medline

Okanoya K (2004) The Bengalese finch: a window on the behavioral neurobiology of birdsong syntax. Ann N Y Acad Sci 1016:724-735. CrossRef Medline

Okanoya K, Yamaguchi A (1997) Adult Bengalese finches (Lonchura striata var. domestica) require real-time auditory feedback to produce normal song syntax. J Neurobiol 33:343-356. Medline

Payne RS, McVay S (1971) Songs of humpback whales. Science 173:585597. CrossRef Medline

Picardo MA, Merel J, Katlowitz KA, Vallentin D, Okobi DE, Benezra SE, Clary RC, Pnevmatikakis EA, Paninski L, Long MA (2016) Population-level representation of a temporal sequence underlying song production in the zebra finch. Neuron 90:866-876. CrossRef Medline

Rosenbaum DA, Kenny SB, Derr MA (1983) Hierarchical control of rapid movement sequences. J Exp Psychol Hum Percept Perform 9:86-102. CrossRef Medline

Sakata JT, Brainard MS (2006) Real-time contributions of auditory feedback to avian vocal motor control. J Neurosci 26:9619-9628. CrossRef Medline

Sakata JT, Brainard MS (2009) Social context rapidly modulates the influence of auditory feedback on avian vocal motor control. J Neurophysiol 102:2485-2497. CrossRef Medline

Schmidt MF (2003) Pattern of interhemispheric synchronization in HVc during singing correlates with key transitions in the song pattern. J Neurophysiol 90:3931-3949. CrossRef Medline

Secora KR, Peterson JR, Urbano CM, Chung B, Okanoya K, Cooper BG (2012) Syringeal specialization of frequency control during song production in the Bengalese finch (Lonchura striata domestica). PLoS One 7:e34135. CrossRef Medline

Sossinka R, Böhner J (1980) Song types in the zebra finch Poephila guttata castanotis. Zeitschrift für Tierpsychologie 53:123-132.

Wang CZ, Herbst JA, Keller GB, Hahnloser RH (2008) Rapid interhemispheric switching during vocal production in a songbird. PLoS Biol 6:e250. CrossRef Medline

Warren TL, Charlesworth JD, Tumer EC, Brainard MS (2012) Variable sequencing is actively maintained in a well learned motor skill. J Neurosci 32:15414-15425. CrossRef Medline

Wild JM (1997) Neural pathways for the control of birdsong production. J Neurobiol 33:653-670. CrossRef Medline

Williams H, Vicario DS (1993) Temporal patterning of song production: participation of nucleus uvaeformis of the thalamus. J Neurobiol 24:903912. CrossRef Medline

Wittenbach JD, Bouchard KE, Brainard MS, Jin DZ (2015) An adapting auditory-motor feedback loop can contribute to generating vocal repetition. PLoS Comput Biol 11:e1004471. CrossRef Medline

Woolley SM, Rubel EW (1997) Bengalese finches Lonchura Striata domestica depend upon auditory feedback for the maintenance of adult song. J Neurosci 17:6380-6390. Medline

Yu AC, Margoliash D (1996) Temporal hierarchical control of singing in birds. Science 273:1871-1875. CrossRef Medline 